\title{
Non-Abelian geometric phase in the diamond nitrogen-vacancy center
}

\author{
Mark A. Kowarsky, ${ }^{1,2,3}$ Lloyd C. L. Hollenberg, ${ }^{1,2}$ and Andrew M. Martin ${ }^{1}$ \\ ${ }^{1}$ School of Physics, The University of Melbourne, Parkville 3010, Australia \\ ${ }^{2}$ Center for Quantum Computation and Communication Technology, School of Physics, The University of Melbourne, \\ Parkville 3010, Australia \\ ${ }^{3}$ Department of Physics, Stanford University, Stanford, California 94305, USA
}

(Received 6 February 2014; published 22 October 2014)

\begin{abstract}
This paper introduces a theoretical framework for understanding the accumulation of non-Abelian geometric phases in rotating nitrogen-vacancy centers in diamond. Specifically, we consider how degenerate states can be achieved and demonstrate that the resulting geometric phase for multiple paths is non-Abelian. We find that the non-Abelian nature of the phase is robust to fluctuations in the path and magnetic field. In contrast to previous studies of the accumulation of Abelian geometric phases for nitrogen-vacancy centers under rotation we find that the limiting time scale is $T_{1}$. As such a non-Abelian geometric phase accumulation in nitrogen-vacancy centers has potential advantages for applications as gyroscopes.
\end{abstract}

DOI: 10.1103/PhysRevA.90.042116

PACS number(s): 06.20.-f, 03.65.Vf, 03.65.Yz, 42.50.Dv

\section{INTRODUCTION}

The geometric phase $[1,2]$ is a fascinating demonstration of how geometry affects quantum systems at the level of Hilbert space structure. Abelian geometric phase arises in nondegenerate systems when the Hamiltonian evolves adiabatically and has been observed in various systems [3-5]. Systems with degenerate energy levels can possess a geometric phase with a non-Abelian structure [6]. This means that different paths of the Hamiltonian produce a geometric phase that in general does not commute, allowing for richer dynamics, providing a platform to implement holonomic quantum computation [7,8], the creation of synthetic fields in quantum gases [9], and the investigation of non-Abelian anyon statistics [10]. Recently an experiment using a superconducting circuit found unambiguous evidence $[11,12]$ of the non-Abelian nature of the phase [13].

In this work, we show that the unique properties afforded to nitrogen-vacancy (NV) defects in diamond enable the interrogation, at room temperature, of the non-Abelian geometric phase, through macroscopic physical rotation of the system. We find that the emergence of non-Abelian geometric phases is robust to magnetic field and path fluctuations. From this we then demonstrate that the accumulation of non-Abelian geometric phase in rotating NV centers can be used as the basis for room temperature gyroscopes with a sensitivity of $\approx 5 \times 10^{-4} \mathrm{rad} / \mathrm{s} / \mathrm{Hz}^{1 / 2}$. This is an order of magnitude larger than the predicted sensitivity of NV gyroscopes, based on Abelian geometric phase accumulation [14,15].

The NV center (for a review, see Ref. [16]) is a defect in diamond whereby a carbon is replaced by a nitrogen and an adjacent carbon is removed. It behaves as an electronic ${ }^{3} A_{2}$ spin triplet system in the ground state, with an excited ${ }^{3} E$ state and a metastable ${ }^{1} A_{1}$ state [see Fig. 1(a)]. A laser with a wavelength shorter than the zero phonon line $(637 \mathrm{~nm})$ polarizes the system into the $m_{s}=0$ ground state and also allows the spin to be read out via the fluorescence intensity. The ground state has relatively long coherence times, even at room temperature, with the inhomogeneous broadening time $T_{2}^{\star}$ of the order of $\mu \mathrm{s}$ and the homogeneous broadening and spin relaxation times $T_{2}$ and $T_{1}$ of the order of $\mathrm{ms}$
[17-19]. The properties afforded to ground state offers a robust and accessible single-spin system with applications in quantum communications [20,21], quantum information [22], nanoscale magnetometry [18,23-29], biosensing [30-34], and thermometry [35-37]. Recently there has been work analyzing the emergence of the Abelian geometric phase in the NV center in rotating systems $[14,15,38]$ which could lead to using them as nanoscale gyroscopes. The ability to manipulate the magnetic sublevels with an external magnetic field enables the possibility of degeneracy between all possible pairs of eigenstates. As such, a single NV system provides an ideal platform to study non-Abelian phases.

A general non-Abelian Berry phase can be understood in terms of a Hamiltonian with $N$ degenerate eigenstates $|a(\vec{\lambda})\rangle$, written in terms of parameters $\vec{\lambda}$ that undergo an adiabatic evolution [11]. For an initial state given by a coherent superposition of degenerate energy eigenstates, the time evolution operator is

$$
U=\mathcal{P} \exp \left(-\int A_{\alpha} d \lambda^{\alpha}\right)
$$

where $\mathcal{P}$ is the path ordering operator and $\alpha$ is summed over the parameters, for example, $\vec{\lambda}=\left(\lambda^{1}, \lambda^{2}\right)=(\theta, \phi)$. The effective gauge potential $A_{\alpha}$, is an $N \times N$ matrix:

$$
A_{a b \alpha}=\left\langle a(\vec{\lambda})\left|\frac{\partial}{\partial \lambda^{\alpha}}\right| b(\vec{\lambda})\right\rangle,
$$

where $a$ and $b$ label the degenerate eigenstates. The effect of $U$ in general will cause a mixing between degenerate eigenstates, and unlike in the nondegenerate $U(1)$ case, the phase cannot be detected directly but only the trace or eigenvalues of $U$ can be determined through a population measurement. To rigorously demonstrate the non-Abelian nature of the acquired phase, two paths in parameter space can be considered (for example those in Fig. 2, labeled 1 and 2), with equal beginning and end points. The non-Abelian nature is seen by comparing $U=$ $U_{2} U_{1}$ with $U^{\prime}=U_{1} U_{2}$. In the Abelian case $U=U^{\prime}$, whereas in the non-Abelian case, in general $U \neq U^{\prime}$. 


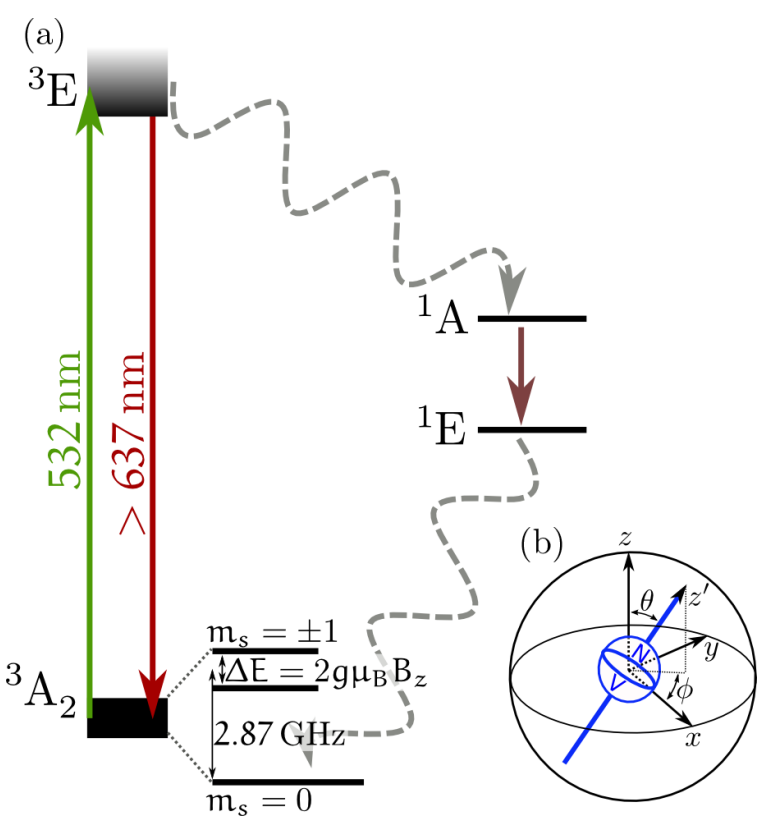

FIG. 1. (Color online) (a) Energy level diagram of the NV center. (b) Geometry of the NV center. Defining the microwave pulses as the $z$ direction, $z^{\prime}$ is the instantaneous direction of the $\mathrm{NV}$ axis, defined with respect to the lab frame, unprimed coordinate system, by $\theta$ and $\phi$.

\section{NON-ABELIAN GEOMETRIC PHASE IN THE NITROGEN-VACANCY CENTER}

The Hamiltonian for the NV spin triplet ground state system in the limit of a low strain diamond and with negligible hyperfine coupling is given by

$$
H^{\prime}=D S_{z^{\prime}}^{2}+\gamma \mathbf{B} \cdot \mathbf{S},
$$

where $z^{\prime}$ is the axis from the nitrogen atom to the adjacent vacancy, the applied magnetic field is $\mathbf{B}$, spin operator $\mathbf{S}$ and $\gamma$ is the gyromagnetic ratio of the NV center,

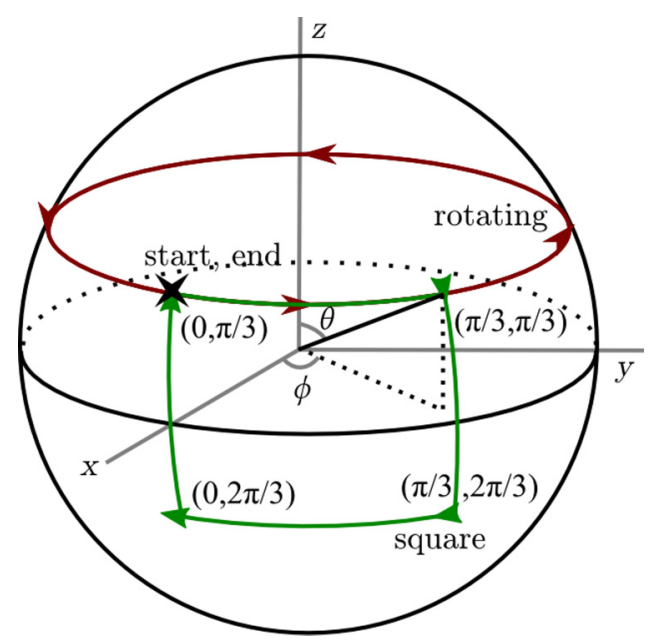

FIG. 2. (Color online) The two paths of rotation of the NV center considered in this paper. Both start and end in the same orientation.
$D \approx 2.87 \mathrm{GHz}$ is the zero-field splitting, and $\hbar=1$. In the lab frame, given by unprimed coordinates, the Hamiltonian takes the form $H=R H^{\prime} R^{-1}$, where $R$ is the rotation operator $R=\exp \left(-i \phi S_{z}\right) \exp \left(-i \theta S_{y}\right) \exp \left(i \phi S_{z}\right)$, where $\theta$ and $\phi$ map between the $z$ and $z^{\prime}$ axes [see Fig. 1(b)]. With no applied field, the $m_{s}= \pm 1$ states are degenerate. Applying a magnetic field along the $z^{\prime}$ axis induces a Zeeman shift of $\Delta E= \pm \gamma B_{z^{\prime}}$. A normalized Hamiltonian will be considered from here on, where $H \rightarrow H / D$ and $\epsilon \equiv \gamma B_{z} / D$ is a measure of the separation of the energy levels. Applying a field of $\epsilon= \pm 1$ makes the $m_{s}=\mp 1$ and $m_{s}=0$ states degenerate.

The effective gauge potential can be calculated for all pairs of states using Eq. (2). For zero applied field, the gauge potential ( $A$ is defined as $\sum_{\alpha} A_{\alpha} d \lambda^{\alpha}$ ) has the following form in the $\{|1\rangle,|-1\rangle\}$ basis:

$$
A=\left(\begin{array}{cc}
-i \cos \theta d \phi & 0 \\
0 & i \cos \theta d \phi
\end{array}\right)
$$

This matrix is Abelian because only entries with $d \phi$ are nonzero. In fact, upon integration around a path, the diagonal entries are proportional to the solid angle enclosed and the phase is identical to the Abelian Berry phase [14,15]. Applying a suitable magnetic field $(\epsilon=\mp 1)$ along the $z^{\prime}$ axis results in a gauge potential in the $\{| \pm 1\rangle,|0\rangle\}$ basis that has a non-Abelian nature, due to the presence of both $d \phi$ and $d \theta$ terms,

$$
A=\left(\begin{array}{cc}
\mp i \cos \theta d \phi & \frac{1}{\sqrt{2}}(i \sin \theta d \phi \mp d \theta) \\
\frac{1}{\sqrt{2}}(i \sin \theta d \phi \pm d \theta) & 0
\end{array}\right)
$$

To unambiguously demonstrate the non-Abelian nature of the phase we first consider a situation when the $|0\rangle$ and $|1\rangle$ states are perfectly degenerate and the paths are exactly those as shown in Fig. 2. To maintain the degeneracy of the $|0\rangle$ and the $|1\rangle$ states, the crystal could be affixed to a magnet that supplies the constant magnetic field such that $\epsilon=-1$. The compound system could then be placed on a spinning device such that the crystal and magnet rotate together and degeneracy is maintained.

Before the whole path is considered, the mixing effect for subpaths is examined by calculating the form of $U$ while holding one of $\theta$ or $\phi$ constant. For $d \phi=0$ the geometric phase in the $\{| \pm 1\rangle,|0\rangle\}$ basis is

$$
\exp \left(-\int_{\Theta} A\right)=\left(\begin{array}{cc}
\cos \left(\frac{\Theta}{\sqrt{2}}\right) & \sin \left(\frac{\Theta}{\sqrt{2}}\right) \\
-\sin \left(\frac{\Theta}{\sqrt{2}}\right) & \cos \left(\frac{\Theta}{\sqrt{2}}\right)
\end{array}\right)
$$

where $\Theta=\int d \theta$ is the polar angle through which the state is rotated. This can be understood in the following manner: A physical rotation of the crystal through an angle of $\Theta$ induces a rotation in the eigenspace of $-\Theta / \sqrt{2}$, independent of $\phi$. In contrast, when $d \theta=0$ the behavior is dependent on $\theta$. Setting $\theta=\pi / 3$ for simplicity and rotating through an azimuthal 
angle $\Phi$ :

$$
\exp \left(-\int_{\Phi} A\right)=e^{i \Phi / 4}\left(\begin{array}{cc}
\cos \left(\frac{\sqrt{7} \Phi}{4}\right)+\frac{i}{\sqrt{7}} \sin \left(\frac{\sqrt{7} \Phi}{4}\right) & -i \sqrt{\frac{6}{7}} \sin \left(\frac{\sqrt{7} \Phi}{4}\right) \\
-i \sqrt{\frac{6}{7}} \sin \left(\frac{\sqrt{7} \Phi}{4}\right) & \cos \left(\frac{\sqrt{7} \Phi}{4}\right)-\frac{i}{\sqrt{7}} \sin \left(\frac{\sqrt{7} \Phi}{4}\right)
\end{array}\right)
$$

Expressing this in terms of the Pauli matrices, Eq. (7) can be thought of as a rotation about the axis that makes the angle $\arctan (1 / \sqrt{6})$ from the negative $x$ - axis to the $z$ - axis. With these two segments of the paths considered, the total effect of the two paths shown in Fig. 2 can be evaluated. The first is a rotation around the sphere at $\theta=\pi / 3$. The phase matrix for this is given by Eq. (7) where $\Phi=2 \pi$. The second path is a square in the space defined by $(\phi, \theta)$. Each leg of the path travels along lines of constant latitude or longitude between the points given by $(\phi, \theta)=\{(0, \pi / 3),(\pi / 3, \pi / 3),(\pi / 3,2 \pi / 3),(0,2 \pi / 3)\}$. Over each of these subpaths the phase accumulated has an Abelian nature, since only one of $d \phi$ or $d \theta$ is nonzero over the length of the path. The path integration can be done analytically, but its form is not concise or enlightening. A numerical approximation of it is

$$
U_{\text {square }} \approx\left(\begin{array}{cc}
0.91+0.23 i & -0.11-0.33 i \\
0.34-0.07 i & 0.66+0.67 i
\end{array}\right) .
$$

Since both paths start and finish at the same point, they offer the potential to show unambiguously the non-Abelian nature of the Berry phase. Initializing the system in the $m_{s}=1$ state and traversing the two paths in one order and measuring the population of the $m_{s}=1$ state, then repeating with the order of the paths interchanged results in a final population difference between the two scenarios of $14.4 \%$. The paths chosen do not optimize the contrast, but do provide a proof of principle realization of the non-Abelian nature of the phase accumulation.

\section{ROBUSTNESS OF NON-ABELIAN GEOMETRIC PHASE TO FLUCTUATIONS}

The analysis above does not deal with experimental considerations such as decoherence, imperfect degeneracies and whether the evolution is adiabatic. To investigate the effect of imperfect degeneracy, the Schrödinger equation $(\hbar=1)$ is written in terms of the the reduced time $s=t / T$, $i \frac{d}{d s}|\psi(s)\rangle=T H(s)|\psi(s)\rangle$, where $T$ is the total time taken for the evolution. The general solution to this equation is $|\psi(s)\rangle=$ $\mathcal{T} \exp \left[-i T \int H(s) d s\right]|\psi(0)\rangle$, where $\mathcal{T}$ is the time-ordering operator. Using the rotating path defined in the previous section (see Fig. 2), this was numerically solved for different values of $T$ and of the energy separation $\Delta=1+\epsilon$ for $\epsilon \approx-1$ (for $\epsilon=$ -1 the $m_{s}=0$ and 1 states are degenerate). These calculations, for $\Phi=2 \pi$ are presented in Fig. 3(a) as a function of rotation time. The maximum rotation time considered is $350 \mu$ s below (above) usual values for $T_{1}\left(T_{2}^{\star}\right)$ of $\mathrm{ms}(\mu \mathrm{s})$ [17-19] and also within potentially achievable $\mathrm{kHz}$ range rotational frequencies. For larger energy separations $\left(\Delta>10^{-4}\right)$ the state quickly reaches the result expected for a nondegenerate adiabatic process. As $\Delta$ is reduced, the first "dip" extends for a longer period, getting closer and closer to the result expected for true degeneracy (solid black line). For a fixed time, the behavior over many orders of magnitude of degeneracy were also considered [see in Fig. 3(b)]. For $|\Delta|<2 \times 10^{-5}$ we find that the population of the $m_{s}=1$ state is within $5 \%$ of the degenerate value (dashed line in Fig. 3), after a complete rotation. This limit scales with evolution time and the zero-field splitting. This enforces the fact that perfect degeneracy is not required, nor are extremely long periods of evolution. All that is required is that the time of evolution is fast compared to the near degeneracy, and slow compared to the third state.
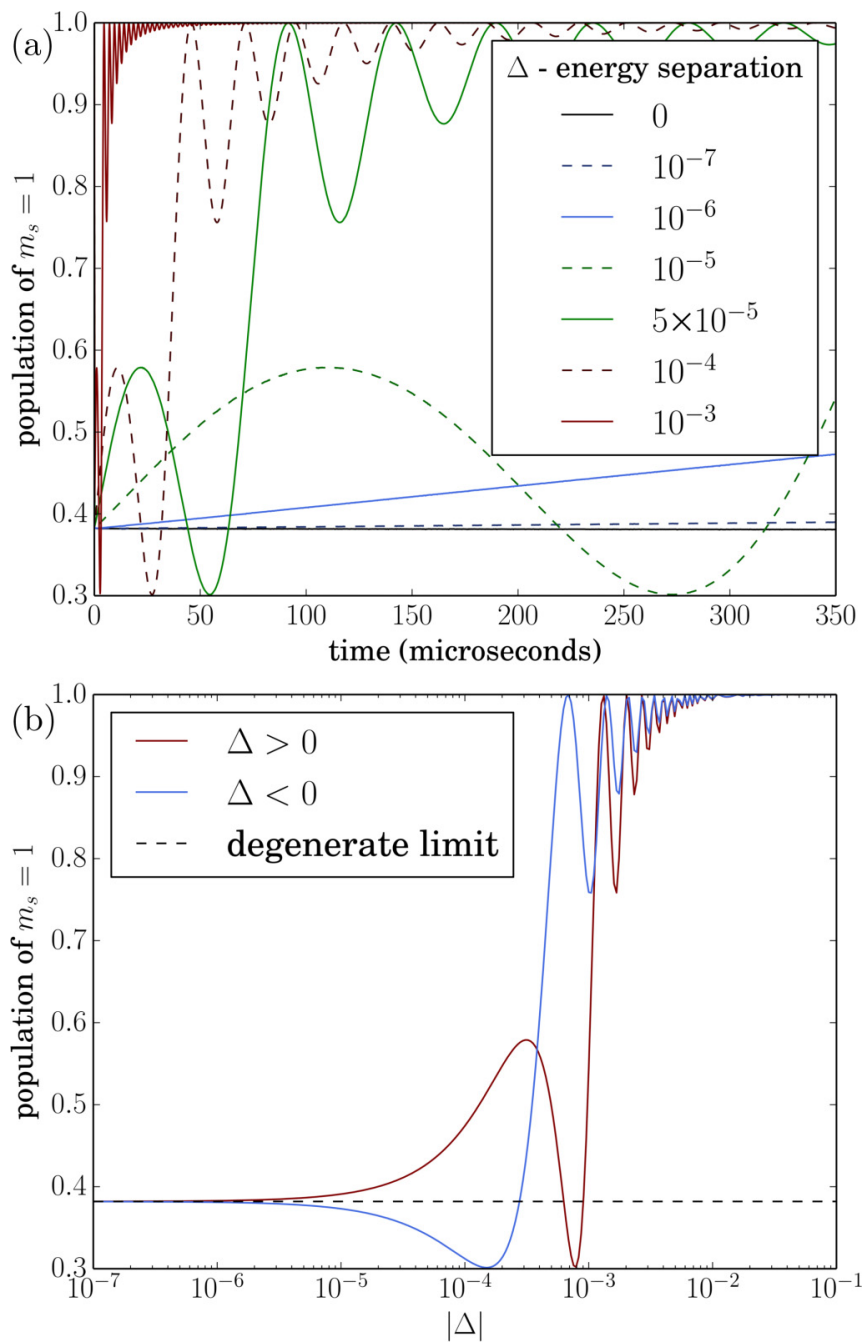

FIG. 3. (Color online) (a) The population in the $m_{s}=1$ state after evolution in the rotating path for varying degrees of degeneracy as a function of rotation time. The asymptotic degenerate result (solid black line) corresponds exactly with the expected adiabatic result. (b) The effect of degeneracy for a rotation of $\approx 3.5 \mu$ s on the population in $m_{s}=1$. The dashed line is the result expected for perfect degeneracy, which is achieved for within $5 \%$ for $|\Delta|<2 \times 10^{-5}$. 


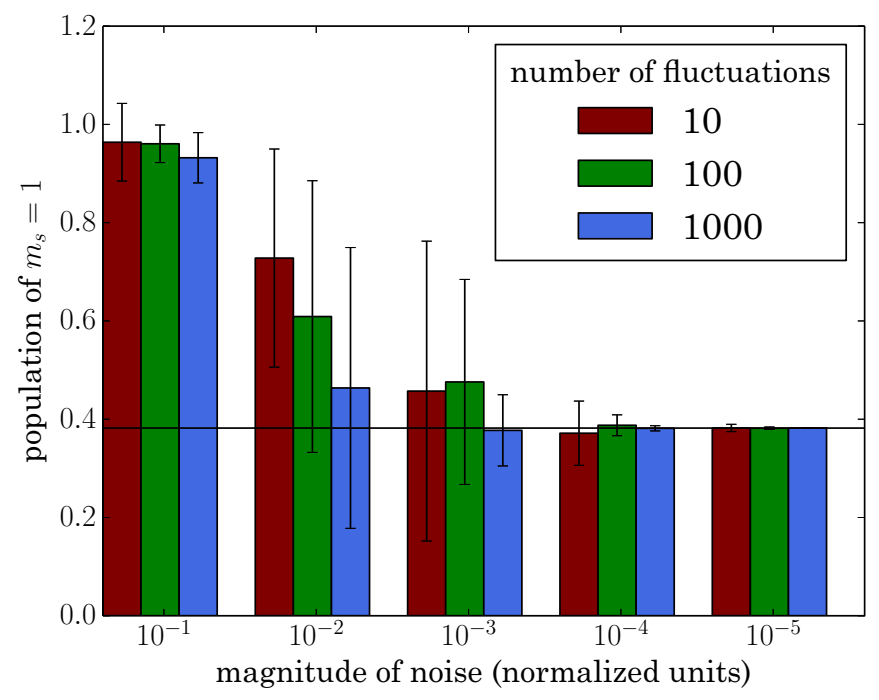

FIG. 4. (Color online) The effect that on-axis magnetic fluctuations, such as those due to $T_{2}$ processes, have on the evolution of the $m_{s}=1$ state after a complete rotation. Error bars are \pm one standard deviation, as determined from simulations. For levels of noise with normalized magnitude below $10^{-4}$ (for $3.5 \mu$ s this is approximately $0.1 \mathrm{G}$ in the NV system), the effects of these fields becomes negligible on the final state population.

Another experimental aspect that needs to be considered is the effect of magnetic field fluctuations. An ensemble of 50 measurements of the same NV system was simulated in the degenerate limit, with Gaussian white noise fluctuations in the field along the NV axis for a range of magnitudes and number of events over the course of the rotating evolution. The results are summarized in Fig. 4. The primary effect of small stray fields is to break the degeneracy and induce a difference in the dynamic phase between the states considered. Since the non-Abelian experiment happens in the regime whereby the nearly degenerate states mix, this additional $U(1)$ phase does not influence the $U(2)$ evolution. This is markedly different to the Abelian experiment where fluctuations in fields increase the variance in the dynamic phase and make the geometric phase harder to recover. This is in line with the fact that $T_{1}$, the spin relaxation time, is the limit for measurements, not $T_{2}^{\star}$ or $T_{2}$ as in the Abelian experimental design.

Besides investigating the effects of a nonideal degeneracy and nonadiabatic motion, we also considered the effects of nonideal paths. A perfectly known path is unobtainable experimentally so in order to determine how errors in the path affect the measurement, perturbations away from the expected polar angle of $\pi / 3$ were simulated for the rotating path. To remain within $5 \%$ of the expected value, the angular divergence required was found to be within $2^{\circ}$. This result was obtained by calculating the population of the state after a single adiabatic rotation at angles $\pi / 3+\varepsilon$ for a range of $\varepsilon$, and requiring that the final population was within $5 \%$ of the $\pi / 3$ result. For the Abelian case the phase accumulated is only dependent on the solid angle enclosed by $z^{\prime}[14]$. As such the Abelian case is robust against path fluctuations [39]. In contrast, for the non-Abelian, changes in the path have the potential to significantly affect the measurement, as different paths mix the states in noncommutative ways.

\section{CONCLUSIONS}

Demonstrating non-Abelian phases in a single NV center experimentally is a worthy goal, but for a single path this approach can be applied to an ensemble of NV centers, in a single rotating crystal, to measure rotation. Consider a system where the $\theta=\pi / 2$, from Eq. (6) after initialization and some rotation, the population remaining in the state and hence the fluorescence will be proportional to $\cos ^{2}(\omega t / \sqrt{2})$, where $\omega$ is the frequency of rotation and $t$ is time. For an ensemble of $N$ centers with a collection efficiency of $\eta$ and contrast of $R$ between the $m_{s}= \pm 1$ and $m_{s}=0$ state, the signal is given by $F=N \eta\left[1-R \sin ^{2}(\omega t / \sqrt{2})\right]$. The smallest detectable frequency is given by $\delta \omega=(d F / d \omega)^{-1} \delta F$, where $\delta F=\sqrt{N \eta}$ is photon shot noise. For a suitable $t$, $d F / d \omega=\sqrt{2} N \eta R t$ and for multiple measurements of time $\tau$ up until the limit $t=T_{1}$, the smallest frequency can be written as

$$
\delta \omega \approx 1 /\left(\alpha R \sqrt{N \eta T_{2}^{\star} \tau}\right),
$$

where $\alpha=\sqrt{2 T_{1} / T_{2}^{\star}}>1$ is the improvement factor over the Abelian scheme $(\alpha \equiv 1)$ which is predicted to have a sensitivity of $5.4 \times 10^{-3} \mathrm{rad} / \mathrm{s} / \mathrm{Hz}^{1 / 2}$ [15], for $R=0.03, \eta=$ $0.5, T_{2}^{\star}=300 \mathrm{~ns}, \tau=0.5 \mathrm{~s}$, and $N=2 \times 10^{14}$. In general, $T_{1}$ is significantly longer than $T_{2}^{\star}$ [17-19] and as such it is predicted that the sensitivity can be improved by an order of magnitude. Comparing with dynamical decoupling schemes [40] the sensitivity for the non-Abelian case is the same order of magnitude. The above neglects the variation in the zero-field splitting, due to local strain, of the NV centers in the crystal. However, this variation is of order $50 \mathrm{kHz}$ [41] corresponding to a variation in $|\Delta| \approx 2 \times 10^{-6}$, which as shown in Fig. 3(b) results in a minimal shift in the measured geometric phase. This discussion has so far focused on the electronic spin, but it should be possible to use the ${ }^{14} \mathrm{~N}$ nuclear spin in a similar fashion as it too is spin-1 [14,15,42]. For the NV center, the required control of the Hamiltonian is carried out by rotating the diamond in physical space. With no applied magnetic field, a Ramsey pulse sequence allows the Abelian phase to be detected. Applying a magnetic field along the NV axis such that the $m_{s}=0$ state is degenerate with one of $m_{s}= \pm 1$ allows the non-Abelian phase to be detected by reading out the population from the spin-dependent fluorescence of the center. From simulations of perturbations to the ideal motion it was found that the non-Abelian phase is robust against decohering effects from magnetic fields. An advantage of the non-Abelian experiment over the Abelian experiment is that the coherence time is increased from $T_{2}^{\star} \rightarrow T_{1}$ and thus the sensitivity to rotations is increased. We have shown that $\mathrm{NV}$ centers may be used as probes for non-Abelian geometric quantum phases, which could allow such measurements of this phase at room temperatures. Additionally, it offers the potential to be a more sensitive gyroscope though further research is needed to resolve signals from multiple axes into the three-axis rotation.

\section{ACKNOWLEDGMENTS}

L.H. and M.K. acknowledge support from the Australian Research Council under the Centre of Excellence scheme 
(Project No. CE110001027). M.K. would like to thank D. Budker and his group for his hospitality and useful discussions relating to extensions to this work during the later stages of the manuscript's preparation.
[1] M. Berry, Proc. R. Soc. London, Ser. A 392, 45 (1984).

[2] B. Simon, Phys. Rev. Lett. 51, 2167 (1983).

[3] J. Anandan, Nature (London) 360, 307 (1992).

[4] P. Leek, J. Fink, A. Blais, R. Bianchetti, M. Göppl, J. Gambetta, D. Schuster, L. Frunzio, R. Schoelkopf, and A. Wallraff, Science 318, 1889 (2007).

[5] M. Möttönen, J. J. Vartiainen, and J. P. Pekola, Phys. Rev. Lett. 100, 177201 (2008).

[6] F. Wilczek and A. Zee, Phys. Rev. Lett. 52, 2111 (1984).

[7] P. Zanardi and M. Rasetti, Phys. Lett. A 264, 94 (1999).

[8] J. Jones, V. Vedral, A. Ekert, and G. Castagnoli, Nature (London) 403, 869 (2000).

[9] J. Dalibard, F. Gerbier, G. Juzeliūnas, and P. Öhberg, Rev. Mod. Phys. 83, 1523 (2011).

[10] C. Nayak, S. Simon, A. Stern, M. Freedman, and S. D. Sarma, Rev. Mod. Phys. 80, 1083 (2008).

[11] A. Zee, Phys. Rev. A 38, 1 (1988).

[12] X. Zhang, Z. Wang, L. Hu, Z. Zhang, and S. Zhu, New J. Phys. 10, 043031 (2008).

[13] A. A. Abdumalikov, Jr., J. M. Fink, K. Juliusson, M. Pechal, S. Berger, A. Wallraff, and S. Filipp, Nature (London) 496, 482 (2013).

[14] D. Maclaurin, M. W. Doherty, L. C. L. Hollenberg, and A. M. Martin, Phys. Rev. Lett. 108, 240403 (2012).

[15] M. P. Ledbetter, K. Jensen, R. Fischer, A. Jarmola, and D. Budker, Phys. Rev. A 86, 052116 (2012).

[16] M. W. Doherty, N. B. Manson, P. Delaney, F. Jelezko, J. Wrachtrup, and L. C. L. Hollenberg, Phys. Rep. 528, 1 (2013).

[17] F. Jelezko, T. Gaebel, I. Popa, A. Gruber, and J. Wrachtrup, Phys. Rev. Lett. 92, 076401 (2004).

[18] J. Taylor, P. Cappellaro, L. Childress, L. Jiang, D. Budker, P. Hemmer, A. Yacoby, R. Walsworth, and M. Lukin, Nat. Phys. 4, 810 (2008).

[19] G. Balasubramanian, P. Neumann, D. Twitchen, M. Markham, R. Kolesov, N. Mizuochi, J. Isoya, J. Achard, J. Beck, J. Tissler et al., Nat. Mater. 8, 383 (2009).

[20] T. Gaebel, I. Popa, A. Gruber, M. Domhan, F. Jelezko, and J. Wrachtrup, New J. Phys. 6, 98 (2004).

[21] A. Beveratos, S. Kühn, R. Brouri, T. Gacoin, J.-P. Poizat, and P. Grangier, Eur. Phys. J. D 18, 191 (2002).

[22] T. Gaebel, M. Domhan, I. Popa, C. Wittmann, P. Neumann, F. Jelezko, J. Rabeau, N. Stavrias, A. Greentree, S. Prawer et al., Nat. Phys. 2, 408 (2006).

[23] G. Balasubramanian, I. Chan, R. Kolesov, M. Al-Hmoud, J. Tisler, C. Shin, C. Kim, A. Wojcik, P. Hemmer, A. Krueger et al., Nature (London) 455, 648 (2008).
[24] J. Maze, P. Stanwix, J. Hodges, S. Hong, J. Taylor, P. Cappellaro, L. Jiang, M. Dutt, E. Togan, A. Zibrov et al., Nature (London) 455, 644 (2008).

[25] C. Degen, Appl. Phys. Lett. 92, 243111 (2008).

[26] J. Cole and L. Hollenberg, Nanotechnology 20, 495401 (2009).

[27] P. Maletinsky, S. Hong, M. S. Grinolds, B. Hausmann, M. D. Lukin, R. L. Walsworth, M. Loncar, and A. Yacoby, Nat. Nanotechnol. 7, 320 (2012).

[28] S. Steinert, F. Ziem, L. Hall, A. Zappe, M. Schweikert, N. Götz, A. Aird, G. Balasubramanian, L. Hollenberg, and J. Wrachtrup, Nat. Commun. 4, 1607 (2013).

[29] H. Mamin, M. Kim, M. Sherwood, C. Rettner, K. Ohno, D. Awschalom, and D. Rugar, Science 339, 557 (2013).

[30] L. McGuinness, Y. Yan, A. Stacey, D. Simpson, L. Hall, D. Maclaurin, S. Prawer, P. Mulvaney, J. Wrachtrup, F. Caruso et al., Nat. Nanotechnol. 6, 358 (2011).

[31] L. Hall, C. Hill, J. Cole, B. Städler, F. Caruso, P. Mulvaney, J. Wrachtrup, and L. Hollenberg, Proc. Natl. Acad. Sci. USA 107, 18777 (2010).

[32] L. T. Hall, G. C. G. Beart, E. A. Thomas, D. A. Simpson, L. P. McGuinness, J. H. Cole, J. H. Manton, R. E. Scholten, F. Jelezko, J. Wrachtrup, S. Petrou, and L. C. L. Hollenberg, Sci. Rep. 2, 401 (2012).

[33] L. Pham, D. Le Sage, P. Stanwix, T. Yeung, D. Glenn, A. Trifonov, P. Cappellaro, P. Hemmer, M. Lukin, H. Park et al., New J. Phys. 13, 045021 (2011).

[34] S. Kaufmann, D. A. Simpson, L. T. Hall, V. Perunicic, P. Senn, S. Steinert, L. P. McGuinness, B. C. Johnson, T. Ohshima, F. Caruso et al., Proc. Natl. Acad. Sci. USA 110, 10894 (2013).

[35] P. Neumann, I. Jakobi, F. Dolde, C. Burk, R. Reuter, G. Waldherr, J. Honert, T. Wolf, A. Brunner, J. H. Shim et al., Nano Lett. 13, 2738 (2013).

[36] D. M. Toyli, F. Charles, D. J. Christle, V. V. Dobrovitski, and D. D. Awschalom, Proc. Natl. Acad. Sci. USA 110, 8417 (2013).

[37] G. Kucsko, P. Maurer, N. Yao, M. Kubo, H. Noh, P. Lo, H. Park, and M. Lukin, Nature (London) 500, 54 (2013).

[38] D. Maclaurin, L. Hall, A. Martin, and L. Hollenberg, New J. Phys. 15, 013041 (2013).

[39] G. De Chiara and G. M. Palma, Phys. Rev. Lett. 91, 090404 (2003).

[40] A. Ajoy and P. Cappellaro, Phys. Rev. A 86, 062104 (2012).

[41] J. S. Hodges, N.Y. Yao, D. Maclaurin, C. Rastogi, M. D. Lukin, and D. Englund, Phys. Rev. A 87, 032118 (2013).

[42] R. H. Clarke, Triplet State ODMR Spectroscopy: Techniques and Applications to Biophysical Systems (Wiley, New York, 1982). 